\title{
An algorithm for piece-wise indefinite quadratic programming problem
}

\author{
Ritu Arora ${ }^{1, *}$ and Kavita Gupta ${ }^{2}$ \\ ${ }^{1}$ Department of Mathematics, Keshav Mahavidyalaya, University of Delhi \\ 110034 Delhi, India \\ E-mail:〈ritunarora@yahoo.in〉 \\ ${ }^{2}$ Department of Mathematics, Kirori Mal College, University of Delhi \\ 110007 Delhi, India \\ E-mail: 〈gupta_kavita31@yahoo.com〉
}

\begin{abstract}
An indefinite quadratic programming problem is a mathematical programming problem which is a product of two linear factors. In this paper, the piece--wise indefinite quadratic programming problem $(P I Q P P)$ is considered. Here, the objective function is a product of two continuous piecewise linear functions defined on a non-empty and compact feasible region. In the present paper, the optimality criterion is derived and explained in order to solve PIQPP. While solving PIQPP, we will come across certain variables which will not satisfy the optimality condition. For these variables, cases have been elaborated so as to move from one basic feasible solution to another till we reach the optimality. An algorithmic approach is proposed and discussed for the PIQPP problem. A numerical example is presented to decipher the tendered method.
\end{abstract}

Keywords: indefinite quadratic programming problem, optimal solution, piece-wise indefinite quadratic function, quasi-concave function

Received: October 10, 2019; accepted: May 26, 2020; available online: July 07, 2020

DOI: $10.17535 /$ crorr.2020.0004

\section{Introduction}

Quadratic programming is an important class of non-linear programming in which the objective function is quadratic and the constraints are linear. Aneja et al. [1] in their paper considered a class of quadratic programs. They considered the maximization and minimization cases of this program assuming the two linear factors to be non-negative. Cabot[3] in his paper considered the problem of maximizing the sum of certain quasi-concave functions over a convex set. Kough [10] developed an algorithm to obtain the global optimum of indefinite quadratic programming problem by employing Benders cut. Pardalos et al. [14] in their paper proposed branch and bound algorithm for finding the global optimum of large scale indefinite quadratic problems over a polytope. Chen and Huang [5] in 2001, proposed a derivative algorithm for solving the inexact quadratic programming which has been a useful tool for environmental system analysis. Shi et al. [15] in 2005 proposed a multiple criteria quadratic programming to classify credit card accounts for business intelligence and decision making.

${ }^{*}$ Corresponding author. 


\subsection{Indefinite quadratic programming problem}

Indefinite quadratic programming problem has diverse applications in material world. It can be seen in production planning, health care, financial planning, corporate planning etc.

Mathematically, the indefinite quadratic programming problem is defined as:

$$
(I Q P P): \operatorname{Max} Z(X)=Z_{1}(X) \cdot Z_{2}(X)=\left(C^{T} X+\alpha\right)\left(D^{T} X+\beta\right)
$$

subject to

$$
\begin{gathered}
A X \leq b \\
X \geq 0
\end{gathered}
$$

where $S_{I}=\{X: A X \leq b ; X \geq 0\}$ is non-empty and compact. $S_{I}$ represents the feasible region of the problem $(P I Q P P)$. Here, $X \in R^{n}$ is a vector of variables; $C, D \in R^{n} ; \alpha, \beta \in R$ and $A \in R^{m \times n}$. Both $Z_{1}(X)$ and $Z_{2}(X)$ are assumed to be positive for all $X \in S_{I}$. Thus, the function $Z(X)$ is both quasi-concave and quasi-convex on $S_{I}$. Hence, $Z(X)$ is explicitly monotone on $S_{I}$. Therefore, the optimal solution to the problem $(I Q P P)$ occurs at an extreme point of $S_{I}$.

\section{2. piece-wise programming problem}

The transmutation of piece-wise linear programming problem to an identical linear programming problem has been proposed by various authors. The proposed methodologies however increase the size of the problem. Fourer [6] in 1985, in his paper developed a simplex algorithm for solving piece-wise linear programming problem. He proved the algorithm on presumption that each piece-wise linear term has finite number of pieces, that a basic feasible starting bases can be found and all bases are non-degenerate. In 1991, Benchekroun and Falk [2] developed an algorithm for non-convex piece-wise linear programs. Fourer [7] in 1992, in his paper provides applications, supporting the practical value of piece-wise linear programming algorithm. In 1994, Murthy and Helgason [12] discussed a specialised direct approach which handles the piece-wise linear structure of the cost function by allowing each arc to have varying costs on different sequences. Chang [4] in 2002, in his paper proposed a modified goal programming technique to solve piece-wise linear functions with $n$ terms. Keha et al. [9] in 2004, formulated linear programs with piece-wise linear objective functions with and without additional binary variables. Problems with piece-wise linear costs give rise to various applications. Ge et al. [8] in 2013, considered the problem of determining the optimal schedules for a given sequence of jobs on a single processor. They extended the result to piece-wise linear cost functions.

In 2007, Pandey and Punen [13] in their paper proposed a simplex algorithm to solve piecewise linear fractional programming problem which is combination of simplex method for linear programs, piece-wise linear programs and the linear fractional programs.

\section{2. piece-wise indefinite quadratic programming problem}

The piece-wise indefinite quadratic programming problem is defined as follows.

$$
\left.\begin{array}{rl}
\text { (PIQPP): } & \operatorname{Max} Z(X)=\sum_{i=1}^{p} Z_{i}\left(x_{i}\right) \\
& \text { subject to } X \in S
\end{array}\right\}
$$

where $S=\left\{X \mid A X=b: 0 \leq x_{i} \leq t_{i} ; i=1,2, \ldots p\right\}$ is the feasible region of the problem $(P I Q P P)$. It is assumed to be non-empty and bounded. Here, $Z_{i}\left(x_{i}\right)=Z_{i 1}\left(x_{i}\right) \cdot Z_{i 2}\left(x_{i}\right)$; $i=1,2, \ldots, p$. 


$$
\begin{gathered}
Z_{i 1}\left(x_{i}\right)=c_{i} x_{i}+\alpha_{i} ; Z_{i 2}\left(x_{i}\right)=d_{i} x_{i}+\beta_{i} ; i=1,2, \ldots, p \\
X=\left(x_{1}, x_{2}, \ldots, x_{p}\right) \in R^{p} ; c_{i}, d_{i} \in R^{p}, i=1,2, . ., p \\
\alpha_{i}, \beta_{i} \in R, A \in R^{n \times p}, \quad b \in R^{n} .
\end{gathered}
$$

Here, each objective function $Z_{i}\left(x_{i}\right) ;(i=1,2,3, \ldots p)$ is the product of two positive valued affine functions, hence, it is quasi-concave. The polyhedron $\mathrm{S}$ defined by the constraint region of the problem $(P I Q P P)$ is assumed to be non-empty and compact. Also, $Z_{i 1}\left(x_{i}\right)$ and $Z_{i 2}\left(x_{i}\right)$ are continuous piece-wise linear functions defined on the feasible region $S$.

The following proposition proves that $Z(X)$ which is the sum of quasi-concave functions is a quasi-concave function on $S$.

Proposition 1. The condition under which the sum of quasi-concave functions is quasi-concave.

Proof. Consider two quasi-concave functions, as

$Z_{1}(X)=\left(c_{1} X+\alpha_{1}\right)\left(d_{1} X+\beta_{1}\right)$ and $Z_{2}(X)=\left(c_{2} X+\alpha_{2}\right)\left(d_{2} X+\beta_{2}\right)$

The sum is defined as $Z(X)=Z_{1}(X)+Z_{2}(X)$.

$Z(X)$ is a quasi-concave function if

$Z\left(\lambda X_{1}+(1-\lambda) X_{2}\right) \geq \operatorname{Min}\left(Z\left(X_{1}\right), Z\left(X_{2}\right)\right), 0 \leq \lambda \leq 1$

Suppose that $\operatorname{Min}\left(Z\left(X_{1}\right), Z\left(X_{2}\right)\right)=Z\left(X_{1}\right)$.

Here, $Z_{1}(X)$ and $Z_{2}(X)$ are two quasi-concave functions.

Therefore, $Z_{1}\left(\lambda X_{1}+(1-\lambda) X_{2}\right) \geq \operatorname{Min}\left(Z_{1}\left(X_{1}\right), Z_{1}\left(X_{2}\right)\right)$

and $Z_{2}\left(\lambda X_{1}+(1-\lambda) X_{2}\right) \geq \operatorname{Min}\left(Z_{2}\left(X_{1}\right), Z_{2}\left(X_{2}\right)\right), 0 \leq \lambda \leq 1$

Case(i): Suppose that $\operatorname{Min}\left(Z_{1}\left(X_{1}\right), Z_{1}\left(X_{2}\right)\right)=Z_{1}\left(X_{1}\right)$ and

$\operatorname{Min}\left(Z_{2}\left(X_{1}\right), Z_{2}\left(X_{2}\right)\right)=Z_{2}\left(X_{1}\right)$.

Consider,

$$
\begin{aligned}
Z\left(\lambda X_{1}+(1-\lambda) X_{2}\right) & =Z_{1}\left(\lambda X_{1}+(1-\lambda) X_{2}\right)+Z_{2}\left(\lambda X_{1}+(1-\lambda) X_{2}\right) \\
& \geq Z_{1}\left(X_{1}\right)+Z_{2}\left(X_{1}\right) \\
& =Z\left(X_{1}\right)
\end{aligned}
$$

Thus, $Z\left(\lambda X_{1}+(1-\lambda) X_{2}\right) \geq \operatorname{Min}\left(Z\left(X_{1}\right), Z\left(X_{2}\right)\right), 0 \leq \lambda \leq 1$.

Hence, $Z(X)$ is a quasi-concave function.

Case(ii): Suppose that $\operatorname{Min}\left(Z_{1}\left(X_{1}\right), Z_{1}\left(X_{2}\right)\right)=Z_{1}\left(X_{1}\right)$

and $\operatorname{Min}\left(Z_{2}\left(X_{1}\right), Z_{2}\left(X_{2}\right)\right)=Z_{2}\left(X_{2}\right)$. Suppose that

$$
Z\left(X_{1}\right)<Z\left(X_{2}\right) \text {, therefore, } \operatorname{Min}\left(Z\left(X_{1}\right), Z\left(X_{2}\right)\right)=Z\left(X_{1}\right)
$$

Consider,

$$
\begin{aligned}
Z\left(\lambda X_{1}+(1-\lambda) X_{2}\right) & =\left(c_{1}\left(\lambda X_{1}+(1-\lambda) X_{2}\right)+\alpha_{1}\right)\left(d_{1}\left(\lambda X_{1}+(1-\lambda) X_{2}\right)+\beta_{1}\right) \\
& +\left(c_{2}\left(\lambda X_{1}+(1-\lambda) X_{2}\right)+\alpha_{2}\right)\left(d_{2}\left(\lambda X_{1}+(1-\lambda) X_{2}\right)+\beta_{2}\right)
\end{aligned}
$$

Take,

$$
\left.\begin{array}{ll}
\alpha_{1}=[\lambda+(1-\lambda)] \alpha_{1} & ; \beta_{1}=[\lambda+(1-\lambda)] \beta_{1} \\
\alpha_{2}=[\lambda+(1-\lambda)] \alpha_{2} & ; \beta_{2}=[\lambda+(1-\lambda)] \beta_{2}
\end{array}\right\}
$$


Put (5) in (4) and on solving, we get

$$
\begin{array}{r}
Z\left(\lambda X_{1}+(1-\lambda) X_{2}\right)=\lambda^{2} Z\left(X_{1}\right)+(1-\lambda)^{2} Z\left(X_{2}\right)+\lambda(1-\lambda)\left[\left(c_{1} X_{1}+\alpha_{1}\right)\left(d_{1} X_{2}+\beta_{1}\right)\right. \\
\left.+\left(c_{1} X_{2}+\alpha_{1}\right)\left(d_{1} X_{1}+\beta_{1}\right)+\left(c_{2} X_{1}+\alpha_{1}\right)\left(d_{2} X_{2}+\beta_{2}\right)+\left(c_{2} X_{2}+\alpha_{2}\right)\left(d_{2} X_{2}+\beta_{2}\right)\right]
\end{array}
$$

Since, $Z\left(X_{2}\right)>Z\left(X_{1}\right)$, therefore,

$$
\begin{aligned}
Z\left(\lambda X_{1}+(1-\lambda) X_{2}\right) & =Z\left(X_{1}\right)+\lambda(1-\lambda)\left[\left(c_{1} X_{1}+\alpha_{1}\right)\left(d_{1} X_{2}+\beta_{1}\right)+\left(c_{1} X_{2}+\alpha_{1}\right)\left(d_{1} X_{1}+\beta_{1}\right)\right. \\
& \left.+\left(c_{2} X_{1}+\alpha_{1}\right)\left(d_{2} X_{2}+\beta_{2}\right)+\left(c_{2} X_{2}+\alpha_{2}\right)\left(d_{2} X_{2}+\beta_{2}\right)-2 Z\left(X_{1}\right)\right]
\end{aligned}
$$

Again, $Z\left(X_{2}\right)>Z\left(X_{1}\right)$, therefore,

$$
\begin{aligned}
\left.\left(c_{1} X_{2}+\alpha_{1}\right)\left(d_{1} X_{2}+\beta_{1}\right)+\left(c_{2} X_{2}+\alpha_{2}\right)\left(d_{2} X_{2}+\beta_{2}\right)\right] & >\left(c_{1} X_{1}+\alpha_{1}\right)\left(d_{1} X_{1}+\beta_{1}\right) \\
& +\left(c_{2} X_{1}+\alpha_{2}\right)\left(d_{2} X_{1}+\beta_{2}\right)
\end{aligned}
$$

Therefore, from (6) we get

$$
\begin{aligned}
Z\left(\lambda X_{1}+(1-\lambda) X_{2}\right) & >Z\left(X_{1}\right)+\left(\frac{c_{1} X_{1}+\alpha_{1}}{d_{1} X_{2}+\beta_{1}}\right)\left(d_{1} X_{2}-d_{1} X_{1}\right)^{2}+\left(\frac{c_{2} X_{1}+\alpha_{2}}{d_{2} X_{2}+\beta_{2}}\right)\left(d_{2} X_{2}-d_{2} X_{1}\right)^{2} \\
& +\left(X_{1}-X_{2}\right)\left(d_{1} \beta_{2}-d_{2} \beta_{1}\right)\left(Z_{2}\left(X_{1}\right)-Z_{2}\left(X_{2}\right)\right)
\end{aligned}
$$

From (3), $Z_{2}\left(X_{1}\right)>Z_{2}\left(X_{2}\right)$, therefore, $Z_{2}\left(X_{1}\right)-Z_{2}\left(X_{2}\right)>0$

$Z\left(\lambda X_{1}+(1-\lambda) X_{2}\right)>Z\left(X_{1}\right)$ if $\left(X_{1}-X_{2}\right)>0,\left(d_{1} \beta_{2}-d_{2} \beta_{1}\right)>0$

or $\left(X_{1}-X_{2}\right)<0,\left(d_{1} \beta_{2}-d_{2} \beta_{1}\right)<0$

that is, if $X_{1}>X_{2}$ then $\frac{d_{1}}{d_{2}}>\frac{\beta_{1}}{\beta_{2}}$ and if $X_{1}<X_{2}$ then $\frac{d_{1}}{d_{2}}<\frac{\beta_{1}}{\beta_{2}}$

In any case, $Z\left(\lambda\left(X_{1}+(1-\lambda) X_{2}\right)>Z\left(X_{1}\right)\right.$.

Hence, $Z(X)$ will be a quasi-concave function.

Similarly, we can derive an expression for the sum of three quasi-concave functions.

\section{Notations}

The breakpoints of $Z_{i 1}\left(x_{i}\right)$ be given by

$0=Z_{i 1}^{0}<Z_{i 1}^{1}<Z_{i 1}^{2}<\ldots<Z_{i 1}^{u_{i}}<Z_{i 1}^{u_{i}+1}=t_{i} ; i=1,2, \ldots, p$

The breakpoints of $Z_{i 2}\left(x_{i}\right)$ be given by

$0=Z_{i 2}^{0}<Z_{i 2}^{1}<Z_{i 2}^{2}<\ldots<Z_{i 2}^{v_{i}}<Z_{i 2}^{v_{i}+1}=t_{i} ; i=1,2, \ldots, p$.

Let $0=\varepsilon_{i}^{0}<\varepsilon_{i}^{1}<\ldots<\varepsilon_{i}^{\lambda_{i}}<\varepsilon_{i}^{\lambda_{i}+1}=t_{i} ;(i=1,2, \ldots, p)$ be the arrangement of distinct elements in an ascending order.

Both the functions $Z_{i 1}^{\ell}\left(i=1,2, \ldots, p ; \ell=0,1, \ldots u_{i}+1\right)$ and $Z_{i 2}^{q}(i=1,2, \ldots ., p$;

$\left.q=0,1, \ldots, v_{i}+1\right)$ are linear within each interval $\left[\varepsilon_{i}^{m}, \varepsilon_{i}^{m+1}\right], m=0,1, \ldots \lambda_{i}$.

Equation (1) can be rewritten as:

$Z_{i 1}\left(x_{i}\right)=c_{i}^{m} x_{i}+\alpha_{i}^{m}, \varepsilon_{i}^{m} \leq x_{i} \leq \varepsilon_{i}^{m+1} ; m=0,1, \ldots . . \lambda_{i} ; i=1,2, \ldots . . p$

$Z_{i 2}\left(x_{i}\right)=d_{i}^{m} x_{i}+\beta_{i}^{m}, \varepsilon_{i}^{m} \leq x_{i} \leq \varepsilon_{i}^{m+1} ; m=0,1, \ldots, \lambda_{i} ; i=1,2, \ldots, p$

$c_{i}^{m}, d_{i}^{m}, \alpha_{i}^{m}, \beta_{i}^{m} \in R ; m=0,1, . ., \lambda_{i} ; i=1,2, . ., p$.

Since $Z_{i 1}\left(x_{i}\right)$ and $Z_{i 2}\left(x_{i}\right)$ are piece--wise continuous linear functions, therefore, we have

$c_{i}^{0} \leq c_{i}^{1} \leq c_{i}^{2} \leq \ldots \leq c_{i}^{\lambda_{i}} ; i=1,2, \ldots, p ; \quad d_{i}^{0} \leq d_{i}^{1} \leq \ldots \leq d_{i}^{\lambda_{i}} ; i=1,2, \ldots, p$

Moreover, the functions are continuous, therefore, we have

$c_{i}^{m} \varepsilon_{i}^{m}+\alpha_{i}^{m}=c_{i}^{m+1} \varepsilon_{i}^{m+1}+\alpha_{i}^{m+1} ; m=0,1, \ldots, \lambda_{i} ; i=1,2, \ldots, p$

and $d_{i}^{m} \varepsilon_{i}^{m}+\beta_{i}^{m}=d_{i}^{m+1} \varepsilon_{i}^{m+1}+\beta_{i}^{m+1} ; m=0,1, \ldots, \lambda_{i} ; i=1,2, \ldots, p$

Using the transformation for piece--wise linear functions [11], let the partition of the variables 
$x_{i}^{\prime}$ s be defined as: $x_{i}=\sum_{m=0}^{\lambda_{i}} y_{m}^{i}$

subject to the constraints

$$
0 \leq y_{m}^{i} \leq \varepsilon_{i}^{m+1}-\varepsilon_{i}^{m}, m=0,1, \ldots, \lambda_{i}
$$

Using (8) in (2), we get

$$
\begin{aligned}
Z_{i 1}\left(x_{i}\right) & =\sum_{i=1}^{p}\left(c_{i} x_{i}+\alpha_{i}\right) \\
& =\sum_{i=1}^{p}\left(c_{i}\left(\sum_{m=0}^{\lambda_{i}} y_{m}^{i}\right)+\alpha_{i}\right) \\
& =\sum_{i=1}^{p} \sum_{m=0}^{\lambda_{i}}\left(c_{i} y_{m}^{i}\right)+\sum_{i=1}^{p} \alpha_{i} \\
\therefore Z_{i 1}\left(x_{i}\right)= & \sum_{i=1}^{p} \sum_{m=0}^{\lambda_{i}}\left(c_{i} y_{m}^{i}\right)+\alpha^{\prime}, \alpha^{\prime}=\sum_{i=1}^{p} \alpha_{i}
\end{aligned}
$$

Similarly, $Z_{i 2}\left(x_{i}\right)=\sum_{i=1}^{p} \sum_{m=0}^{\lambda_{i}}\left(d_{i} y_{m}^{i}\right)+\beta^{\prime}, \beta^{\prime}=\sum_{i=1}^{p} \beta_{i}$.

Also, $A X=b \Rightarrow \sum_{i=1}^{p} \sum_{m=0}^{\lambda_{i}} a_{h_{i}} y_{m}^{i}=b_{h}, h=1, \ldots, m$

$0 \leq y_{m}^{i} \leq \varepsilon_{i}^{m+1}-\varepsilon_{i}^{m} ; m=0,1, \ldots, \lambda_{i} ; i=1,2, . ., p$.

Using the transformation from equation (8) in equation (2), the result is equation (9). Thus, using equation (9), problem (1) is transformed to the following problem,

$\operatorname{Max} Z(X)=\sum_{i=1}^{p} Z_{i}\left(x_{i}\right)=\sum_{i=1}^{p} Z_{i 1}\left(x_{i}\right) \cdot Z_{i 2}\left(x_{i}\right)$

subject to

$\sum_{i=1}^{p} \sum_{m=0}^{\lambda_{i}} a_{h_{i}} y_{m}^{i}=b_{h}, \quad h=1, \ldots, m$

$0 \leq y_{m}^{i} \leq \varepsilon_{i}^{m+1}-\varepsilon_{i}^{m} ; m=0,1, \ldots, \lambda_{i} ; i=1,2, . ., p$.

The optimal solution for this problem exists as this is an indefinite quadratic programming problem. Solving this problem by its conventional method increases the size of the problem. Thus, arises the need of developing an algorithm to obtain the solution of (PIQPP).

\section{Methodology to solve $(P I Q P P)$}

The piece-wise indefinite quadratic programming problem $(P I Q P P)$ is defined as:

$\operatorname{Max} Z(X)=\sum_{i=1}^{p} Z_{i}\left(x_{i}\right)$

subject to

$A X=b$

$X \in S$

$0 \leq x_{i} \leq t_{i}, i=1,2,3, \ldots . p$.

Here, we have $A X=B$

This can be written as $B^{*} X_{B^{*}}+N^{*} X_{N^{*}}=b$

$\Rightarrow X_{B^{*}}=B^{-1}\left(b-N^{*} X_{N^{*}}\right)$

$\Rightarrow X_{B^{*}}=B^{-1} b-\sum_{z \in N^{*}} B^{-1} A_{i} x_{i}$

Here, $B^{*}$ is the $n \times n$ basis matrix and $X_{B^{*}}$ is the basic feasible solution corresponding to the basis $B^{*} . N^{*}$ is the non-basis matrix defined as $N^{*}=\left\{i: a_{i} \notin B^{*} ; x_{i} \in\left(\varepsilon_{i}^{0}, \varepsilon_{i}^{1}, \ldots, \varepsilon_{i}^{\lambda_{i+1}}\right)\right\}$.

The non-basic matrix $N^{*}$ is further decomposed into $\bar{N}^{*}$ where, $\bar{N}^{*}=\left\{i: i \in N^{*}, x_{i}=\varepsilon_{i}^{\delta_{i}}\right.$ for some $\left.\delta_{i} \in\left(0,1, \ldots, \lambda_{i+1}\right)\right\}$.

The basis structure for the problem $(P I Q P P)$ is $\left(B^{*}, N^{*}, \bar{N}^{*}\right)$. 
Let $X^{*}=\left(X_{B^{*}}, X_{N^{*}}\right)$ be a basic feasible solution corresponding to this basis structure. Each non-basic variable $x_{i}$ will take value at one of the break points of $Z_{i 1}\left(x_{i}\right)$ or $Z_{i 2}\left(x_{i}\right)$.

Let $\mu_{B_{j}^{*}}$ be the index such that $\varepsilon_{B_{j}^{*}}^{\mu_{B_{j}^{*}}} \leq x_{B_{j}^{*}} \leq \varepsilon_{B_{j}^{*}}^{\mu_{B_{j}^{*}+1}}, j=1,2, \ldots, n$.

Definition 1. $Z_{1 j}$-slope vector: The $n-$ vector $c_{B^{*}}$ whose $j^{\text {th }}$ coordinate is $c_{B_{j}^{*}}^{\mu_{B_{j}^{*}}}$ corresponding to the basis structure $\left(B^{*}, N^{*}, \bar{N}^{*}\right)$ is called $Z_{1 j}$ - slope vector.

Definition 2. $Z_{2 j}$-slope vector: The n-vector $d_{B^{*}}$ whose $j^{\text {th }}$ coordinate is $d_{B_{j}^{*}}^{\mu_{B_{j}^{*}}}$ corresponding to the basis structure $\left(B^{*}, N^{*}, \bar{N}^{*}\right)$ is called $Z_{2 j}$ - slope vector.

Result 1: Correspondence between basic feasible solution to $(I Q P P)$ and an optimal solution to $(P I Q P P)$ exists.

Proof. Let $X^{\prime}=\left(x_{1}^{\prime}, x_{2}^{\prime}, \ldots, x_{p}^{\prime}\right) \in R^{p}$ be an optimal solution to $(P I Q P P)$. Choose an index $i_{k}$ for each $i=1,2, \ldots . p$ such that $\varepsilon_{i}^{i_{k}} \leq x_{i}^{\prime} \leq \varepsilon_{i}^{i_{k}+1}$. Then we have,

$$
\begin{array}{r}
\operatorname{Max} Z\left(X^{\prime}\right)=\sum_{i=1}^{p} Z_{i}\left(X^{\prime}\right) \\
\text { subject to } A X^{\prime}=b
\end{array}
$$

where $Z_{i 1}\left(X^{\prime}\right)=\sum_{i=1}^{p} c_{i} x_{i}^{\prime}+\alpha ; Z_{i 2}\left(X^{\prime}\right)=\sum_{i=1}^{p} d_{i} x_{i}^{\prime}+\beta ; \alpha=\sum_{i=1}^{p} \alpha_{i} ; \beta=\sum_{i=1}^{p} \beta_{i}$

$$
\varepsilon_{i}^{i_{k}} \leq x_{i}^{\prime} \leq \varepsilon_{i}^{i_{k}+1}, i=1,2, \ldots, p .
$$

Thus, if the set of feasible solutions of $(I Q P P)$ is non-empty and the objective function is positive for all the feasible solutions and the values of the variables lies within the specified bounds (as in (10)), then an optimal solution to $(P I Q P P)$ is a basic feasible solution to (IQPP).

\subsection{Optimality condition}

A non-basic variable $x_{i}$ changes its value from its current breakpoint value either in the left side direction or in the right side direction. The left hand side reduced cost is denoted by $\xi_{i}^{-}$and the right hand side reduced cost is denoted by $\xi_{i}^{+}$. The left hand side reduced cost is defined as:

$$
\begin{aligned}
\xi_{i}^{-} & =Z_{1}\left(d_{B^{*}} B^{*-1} A_{i}-d_{i}^{\delta_{i}-1}\right)+Z_{2}\left(c_{B^{*}} B^{*-1} A_{i}-c_{i}^{\delta_{i}-1}\right) \\
& -\theta_{i}\left(d_{B^{*}} B^{*-1} A_{i}-d_{i}^{\delta_{i}-1}\right)\left(c_{B^{*}} B^{*-1} A_{i}-c_{i}^{\delta_{i}-1}\right)
\end{aligned}
$$

The right hand side reduced cost is defined as:

$$
\begin{aligned}
\xi_{i}^{+} & =Z_{1}\left(d_{B^{*}} B^{*-1} A_{i}-d_{i}^{\delta_{i}}\right)+Z_{2}\left(c_{B^{*}} B^{*-1} A_{i}-c_{i}^{\delta_{i}}\right) \\
& -\theta_{i}\left(d_{B^{*}} B^{*-1} A_{i}-d_{i}^{\delta_{i}}\right)\left(c_{B^{*}} B^{*-1} A_{i}-c_{i}^{\delta_{i}}\right)
\end{aligned}
$$

Here, $Z_{1}\left(=\sum_{i=1}^{p} Z_{i 1}\right)$ and $Z_{2}\left(=\sum_{i=1}^{p} Z_{i 2}\right)$ are the values of the objective functions at the current basic feasible solution $X^{*}=\left(X_{B^{*}}, X_{N^{*}}\right) . c_{B^{*}} ; d_{B^{*}}$ are the corresponding $Z_{1 j}$ and $Z_{2 j}$ slope vector respectively.

If $\delta_{i}=\lambda_{i+1}$ then $\xi_{i}^{+}=0$. If $\delta_{i}=0$ then $\xi_{i}^{-}=0$.

For all the basic variables, $x_{i}, \xi_{i}^{-}=\xi_{i}^{+}=0$ 
Theorem 1. A non-degenerate basic feasible solution is optimal for (PIQPP) if and only if $\xi_{i}^{-} \leq 0, \xi_{i}^{+} \geq 0, i=1,2, \ldots, p$.

Proof. Let $B^{*}$ be the $n \times n$ basis matrix and let $N^{*}$ be the non-basic matrix. The objective function values corresponding to the basis $B^{*}$ are given by

$Z_{i 1}=\left(C_{B^{*}}\right)_{i} B^{*-1} b-\sum_{j \in N^{*}}\left(z_{j}^{i 1}-c_{j}\right) x_{j}^{*}+\alpha_{i}, i=1,2, \ldots, p$

$Z_{i 2}=\left(D_{B^{*}}\right)_{i} B^{*-1} b-\sum_{j \in N^{*}}\left(z_{j}^{i 2}-d_{j}\right) x_{j}^{*}+\beta_{i}, i=1,2, \ldots, p$

Suppose that $X_{B^{*}}^{0}$ be a non-degenerate optimal solution for $(P I Q P P)$ corresponding to the basis $B^{*}$, where $x_{j}^{0}=\varepsilon_{j}^{\lambda_{j}}, j \in N^{*}$. Therefore, improved objective function values are given by $Z_{i 1}\left(X_{B^{*}}^{0}\right)=\left(C_{B^{*}}\right)_{i} B^{*-1} b-\sum_{j \in N^{*}}\left(z_{j}^{i 1}-c_{j}\right) \varepsilon_{j}^{\lambda_{j}}+\alpha_{i}, i=1, \ldots, p$

$Z_{i 2}\left(X_{B^{*}}^{0}\right)=\left(D_{B^{*}}\right)_{i} B^{*-1} b-\sum_{j \in N^{*}}\left(z_{j}^{i 2}-d_{j}\right) \varepsilon_{j}^{\lambda_{j}}+\beta_{i}, i=1, \ldots, p$

and $Z_{i}\left(X_{B^{*}}^{0}\right)=Z_{i 1}\left(X_{B^{*}}^{0}\right) \cdot Z_{i 2}\left(X_{B^{*}}^{0}\right) ; i=1,2, \ldots, p$.

Suppose that $\xi_{r}^{+}<0$ corresponding to some non-basic variable $x_{r}^{*}$. In order to find a new feasible solution, let the non-basic variable $x_{r}^{*}$ undergoes a change $\phi_{r}^{*}$. Let the new feasible solution be given by $\hat{X}^{*}=\left(\hat{x}_{i}\right)^{*}$ where,

$$
\left(\hat{x}_{i}\right)^{*}= \begin{cases}\varepsilon_{r}^{\lambda_{r}}+\phi_{r}^{*}, & j=r \\ \varepsilon_{j}^{\lambda_{j}}, & j \in N \backslash\{r\}\end{cases}
$$

The objective function value corresponding to a new feasible solution $\hat{X}^{*}$ given by

$$
\begin{aligned}
& Z_{i 1}\left(\hat{X}^{*}\right)=C_{\hat{B}_{i}}\left(\hat{B}^{-1} b\right)-\sum_{j \in N \backslash\{r\}}\left(z_{j}^{i 1}-c_{j}\right) \varepsilon_{j}^{\lambda_{j}}-\left(z_{r}^{i 1}-c_{r}\right)\left(\varepsilon_{r}^{\lambda_{r}}+\phi_{r}^{*}\right)+\alpha_{i}, i=1, \ldots, p \\
& =C_{\hat{B}_{i}}\left(\hat{B}^{-1} b\right)-\left[\sum_{j \in N \backslash\{r\}}\left(z_{j}^{i 1}-c_{j}\right) \varepsilon_{j}^{\lambda_{j}}+\left(z_{r}^{i 1}-c_{r}\right) \varepsilon_{r}^{\lambda_{r}}\right]-\left(z_{r}^{i 1}-c_{r}\right) \phi_{r}^{*}+\alpha_{i}, i=1, \ldots, p \\
& =\left[C_{\hat{B}_{i}}\left(\hat{B}^{-1} b\right)-\sum_{j \in N}\left(z_{j}^{i 1}-c_{j}\right) \varepsilon_{j}^{\lambda_{j}}+\alpha_{i}\right]-\phi_{r}^{*}\left(z_{r}^{i 1}-c_{r}\right) ; i=1, \ldots, p \\
& =Z_{i 1}\left(X^{0}\right)^{*}-\varphi_{r}^{*}\left(z_{r}^{i 1}-c_{i}\right) ; i=1, \ldots, p .
\end{aligned}
$$

Therefore,

$$
Z_{i 1}(\hat{X})^{*}=Z_{i 1}\left(X^{0}\right)^{*}-\phi_{r}^{*}\left(z_{r}^{i 1}-c_{r}\right) ; i=1,2, \ldots p .
$$

Similarly, $Z_{i 2}\left(X^{*}\right)=Z_{i 2}\left(X^{0}\right)^{*}-\phi_{r}^{*}\left(z_{r}^{i 2}-d_{r}\right)$.

Therefore, $Z_{i}(\hat{X})^{*}=Z_{i 1}(\hat{X})^{*} \cdot Z_{i 2}(\hat{X})^{*}$

Now, $Z_{i}(\hat{X})^{*}-Z_{i}\left(X^{0}\right)^{*}=\left[Z_{i 1}\left(X^{0}\right)^{*}-\phi_{r}^{*}\left(z_{r}^{i 1}-c_{r}\right)\right]\left[Z_{i 2}\left(X^{0}\right)^{*}-\phi_{r}^{*}\left(z_{r}^{i 2}-d_{r}\right)\right]-Z_{i}\left(X^{0}\right)^{*}$

$=Z_{i 1}\left(X^{0}\right)^{*} Z_{i 2}\left(X^{0}\right)^{*}-Z_{i 1}\left(X^{0}\right)^{*} \phi_{r}^{*}\left(z_{r}^{i 2}-d_{r}\right)-Z_{i 2}\left(X^{0}\right)^{*}\left(z_{r}^{i 1}-c_{r}\right)+\phi_{r}^{* 2}\left(z_{r}^{i 1}-c_{r}\right)\left(z_{r}^{i 2}-d_{r}\right)-$ $Z_{i 1}\left(X^{0}\right)^{*} Z_{i 2}\left(X^{0}\right)^{*}$

$=-\varphi_{r}^{*}\left[Z_{i 1}\left(X^{0}\right)^{*}\left(z_{r}^{i 2}-d_{r}\right)+Z_{i 2}\left(X^{0}\right)^{*}\left(z_{r}^{i 1}-c_{r}\right)-\varphi_{r}^{*}\left(z_{r}^{i 2}-d_{r}\right)\left(z_{r}^{i 1}-c_{r}\right)\right]$

Thus,

$$
Z_{i}(\hat{X})^{*}-Z_{i}\left(X^{0}\right)^{*}=-\phi_{r}^{*} \xi_{r}^{+}
$$

Now, $\xi_{r}^{+}<0 ; \varphi_{r}^{*}>0$, we get $Z_{i}(\hat{X})^{*}-Z_{i}\left(X^{0}\right)^{*}>0$ or $Z_{i}(\hat{X})^{*}>Z_{i}\left(X^{0}\right)^{*}$

This implies $\left(X^{0}\right)^{*}$ is not an optimal solution for $(P I Q P P)$ which is a contradiction to our assumption. Thus, $\xi_{r}^{+}>0$ is an optimality condition for $(P I Q P P)$. Similarly, case can be discussed where $\xi_{r}^{-} \leq 0$.

Conversely, suppose that $X^{*}=\left(X_{B^{*}}, X_{N^{*}}\right)$ be a non-degenerate basic feasible solution to $(P I Q P P)$ for which $\xi_{r}^{-} \leq 0$ and $\xi_{r}^{+} \geq 0$. From equation (14), we have

$Z_{i}(\hat{X})^{*}-Z_{i}\left(X^{0}\right)^{*}=-\phi_{r}^{*} \xi_{r}^{+}$

Since, $\phi_{r}^{*}>0$ and $\xi_{r}^{+} \geq 0$, therefore, $Z_{i}(\hat{X})^{*}-Z_{i}\left(X^{0}\right)^{*} \leq 0$ or $Z_{i}(\hat{X})^{*} \leq Z_{i}\left(X^{0}\right)^{*}$

Thus, $\left(X^{0}\right)^{*}$ is an optimal solution to $(P I Q P P)$. 


\subsection{How to move from one basic feasible solution to another improved basic feasible solution}

Let $X_{B^{*}}^{0}$ be the current basic feasible solution corresponding to the basis $B^{*}$. If corresponding to this basis $\xi_{i}^{-} \leq 0$ and $\xi_{i}^{+} \geq 0, \forall i=1, \ldots, p$ then $X_{B^{*}}^{0}$ is an optimal solution to $(P I Q P P)$.

Suppose there exists at least one non-basic variable $x_{r}$, corresponding to which optimality condition is not satisfied, that is, either $\xi_{r}^{-}>0$ or $\xi_{r}^{+}<0$. Hence, the variable $x_{r}$ will be the entering variable. If $\xi_{r}^{-}>0$, then in order to improve the solution, the value of the variable $x_{r}$ can be reduced from $\xi_{r}^{\lambda_{r}}$ to $\xi_{r}^{\lambda_{r}}-\varphi_{r}^{*}$, keeping the values of all other non-basic variables at their current breakpoint. Similarly, if $\xi_{r}^{+}<0$, then $x_{r}$ can be increased from $\varepsilon_{r}^{\lambda_{r}}$ to $\varepsilon_{r}^{\lambda_{r}}+\varphi_{r}^{*}$, to obtain an improved solution. This is discussed in following cases-

Case 1: Let $\varepsilon_{r}^{+}<0$ and $x_{r}$ be the corresponding entering variable. In this case, the non-basic variable $x_{r}$ undergoes a change $\varphi_{r}^{*}$. Let the new solution so obtained be $\left(\hat{X}_{r}\right)^{*}=\left(\hat{x}_{r}\right)$, where

$$
\left.\begin{array}{l}
\left(\hat{x}_{j}\right)_{r}=\left(x_{j}^{*}\right)_{r}-y_{j r}^{*} \varphi_{r}^{*}, \forall j \in I^{*} \\
\left(\hat{x}_{r}\right)=x_{r}^{0}+\phi_{r}^{*} \\
\left(\hat{x}_{j}\right)_{r}=\left(x_{j}^{0}\right), j \in N^{*} \backslash\{r\}
\end{array}\right\}
$$

Here, $I^{*}=\left\{j: a_{j} \in B^{*}\right\}$.

The value of $\varphi_{r}^{*}$ should be restricted so that $\varepsilon_{B^{*}}^{\mu\left(B^{*}\right)} \leq x_{B^{*}} \leq \varepsilon_{B^{*}}^{\mu\left(B^{*}\right)+1}$ and $\hat{x}_{r} \leq \varepsilon_{r}^{\lambda_{r}+1}$.

The new solution $\left(\hat{X}_{r}\right)^{*}$ is a feasible extreme point, provided $\phi_{r}^{*}=\min \left\{\eta_{1}, \eta_{2}, \eta_{3}\right\}$ where

$$
\begin{aligned}
& \eta_{1}=\min \left\{\frac{x_{B_{j}^{*}}-\varepsilon_{B_{j}^{*}}^{\mu\left(B_{j}^{*}\right)+1}}{y_{j r}^{*}} \mid y_{j r}^{*}<0\right\} \\
& \eta_{2}=\min \left\{\frac{\left.x_{B_{j}^{*}-\varepsilon_{B_{j}^{*}}^{\mu\left(B_{j}^{*}\right)}} \mid y_{j r}^{*}>0\right\}}{y_{j r}^{*}} \mid\right. \\
& \eta_{3}=\min \left\{\varepsilon_{r}^{\lambda_{r}+1}-\varepsilon_{r}^{\lambda_{r}}\right\}
\end{aligned}
$$

Since the basic feasible solution is non-degenerate, $\eta_{1}, \eta_{2}, \eta_{3}$ are all distinct.

Case (i): Let $\phi_{r}^{*}=\eta_{1}$ that is, $\phi_{r}^{*}=\left(\frac{x_{B_{s}^{*}-\varepsilon_{B_{s}^{*}}^{\mu}\left(B_{s}^{*}+1\right)}}{y_{s r}^{*}}\right)$ for some $s_{r} \in I^{*}$. This implies that $x_{B_{r}^{*}}$ becomes basic and $x_{B_{s}^{*}}$ departs from the basis and attains the value at the break point $\varepsilon_{B_{s}^{*}}^{\mu_{\left(B_{s}^{*}+1\right)}}$. The change in the values of the objective functions $Z_{i 1}(\hat{X})^{*}, Z_{i 2}(\hat{X})^{*}$ and the basic variables are given by the equations (13) and (15), respectively. The $r^{t h}$ component of $C_{B^{*}}$ and $D_{B^{*}}$

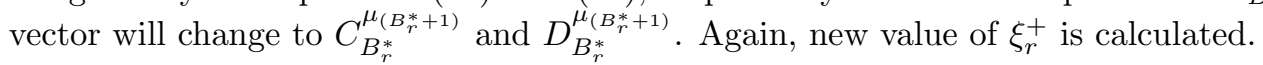

If $\xi_{r}^{+} \geq 0$, then $\varphi_{r}^{*}$ is the maximum possible change in the value of the objective functions, given by equation (14). If $\xi_{r}^{+}<0$, then there is a possibility of further increasing the value of $\varphi_{r}^{*}$ by redefining $\mu_{B_{s}^{*}}$ as $\mu_{B_{s}^{*}+1}$ in $\eta_{1}$. This generates new value of $\varphi_{r}^{*}$. The process is repeated until optimality condition is attained.

Case (ii): Let $\varphi_{r}^{*}=\eta_{2}$, that is $\phi_{r}^{*}=\left(\frac{x_{B_{s}^{*}-\varepsilon_{B_{s}^{*}}^{\mu}\left(B_{s}^{*}\right)}}{y_{s r}^{*}}\right)$ for some $s_{r} \in I^{*}$.

This implies that $x_{B_{r}^{*}}$ becomes basic and $x_{B_{s}^{*}}$ departs from the basis. The change in the values of the objective functions and the basic variables are given by equations (13) and (15), respectively. The $r^{t h}$ component of $C_{B^{*}}$ and $D_{B^{*}}$ will change to $C_{B_{r}^{*}}^{\mu_{\left(B_{r}^{*}\right)}-1}$ and $D_{B_{r}^{*}}^{\mu_{\left(B_{r}^{*}\right)}-1}$ respectively.

If $\xi_{r}^{+} \geq 0$, then the optimal solution is attained. If $\xi_{r}^{+}<0$, then change the value of $\varphi_{r}^{*}$ by redefining $\mu_{B_{s}^{*}}$ as $\mu_{B_{s}^{*}-1}$ in $\eta_{2}$. A new value of $\varphi_{r}^{*}$ is generated and the process is repeated.

Case (iii): Let $\phi_{r}^{*}=\eta_{3}$, that is, $\phi_{r}^{*}=\left(\varepsilon_{r}^{\lambda_{r}+1}-\varepsilon_{r}^{\lambda_{r}}\right)$. This means that $x_{B_{r}^{*}}$ remains non-basic 
and the $r^{\text {th }}$ component of the set $\bar{N}^{*}$ will change to $\delta_{r}+1$. Also, $c_{i}^{\delta_{i}}$ and $d_{i}^{\delta_{i}}$ will change to $c_{i}^{\delta_{i}+1}$ and $d_{i}^{\delta_{i}+1}$ respectively. The change in the values of the objective functions and basic variables are given by equations (13) and (15), respectively.

If $\xi_{r}^{+} \geq 0$, then $\varphi_{r}^{*}$ is the maximum possible change in the value (14) and (15).

If $\xi_{r}^{+}<0$, then $\varphi_{r}^{*}$ can be calculated by redefining $\delta_{r}$ as $\delta_{r}+1$. Thus, the process is repeated until optimality condition is obtained.

Case II: Let $\xi_{r}^{-}>0$ and $x_{r}$ be the corresponding entering variable. If the variable $x_{B_{r}^{*}}=\varepsilon_{r}^{\lambda_{r}}$ undergoes a change, then the new solution is given by $\left(\hat{X}_{r}\right)^{*}=\left(\hat{x}_{r}\right)$, where,

$$
\left.\begin{array}{l}
\left(\hat{x}_{j}\right)_{r}=\left(x_{j}^{*}\right)_{r}+y_{j r}^{*} \phi_{r}^{*}, \forall j \in I^{*} \\
\left(\hat{x}_{r}\right)=x_{r}^{0}+\phi_{r}^{*} \\
\left(\hat{x}_{j}\right)_{r}=x_{j}^{0}, j \in N^{*} \backslash\{r\}
\end{array}\right\}
$$

The objective function value corresponding to new feasible solution $\left(\hat{X}_{r}\right)^{*}$ is given by

$$
Z_{i}(\hat{X})^{*}=\left[Z_{i 1}\left(X^{0}\right)^{*}+\phi_{r}^{*}\left(z_{r}^{i 1}-c_{r}\right)\right]\left[Z_{i 2}\left(X^{0}\right)^{*}+\phi_{r}^{*}\left(z_{r}^{i 2}-d_{r}\right)\right]
$$

The value of $\varphi_{r}^{*}$ should be restricted so that $\varepsilon_{B^{*}}^{\mu\left(B^{*}\right)} \leq x_{B^{*}} \leq \varepsilon_{B^{*}}^{\mu\left(B^{*}\right)+1}$ and $\hat{x}_{r} \geq \varepsilon_{r}^{\lambda_{r}-1}$. The new solution is a feasible extreme point, provided $\phi_{r}^{*}=\min \left\{\eta_{1}, \eta_{2}, \eta_{3}\right\}$, where

$$
\left.\begin{array}{l}
\eta_{1}=\min \left\{\frac{x_{B_{j}^{*}}-\varepsilon_{B_{j}^{*}}^{\mu\left(B_{j}^{*}\right)+1}}{y_{j r}^{*}} \mid y_{j r}^{*}<0\right\} \\
\eta_{2}=\min \left\{\frac{x_{B_{j}^{*}}-\varepsilon_{B_{j}^{*}}^{\mu\left(B^{*}\right)}}{y_{j r}^{*}} \mid y_{j r}^{*}>0\right\} \\
\eta_{3}=\min \left\{\varepsilon_{r}^{\lambda_{r}}-\varepsilon_{r}^{\lambda_{r}-1}\right\}
\end{array}\right\}
$$

Since the basic feasible solution is non-degenerate, therefore, $\eta_{1}, \eta_{2}, \eta_{3}$ are all distinct.

If $\phi_{r}^{*}=\eta_{1}$, then the basic variable say $x_{B_{s}^{*}}$ reaches the breakpoint $\varepsilon_{B_{s}}^{\mu\left(B_{s}\right)+1}$.

If $\phi_{r}^{*}=\eta_{2}$, then the basic variable say $x_{B_{s}^{*}}$ reaches the breakpoint value $\varepsilon_{B_{s}}^{\mu\left(B_{s}\right)}$.

If $\phi_{r}^{*}=\eta_{3}$, then the non-basic variable say $x_{r}$ moves to the new break point value.

The change in the values of the basic variables and the objective functions due to change in $\varphi_{r}^{*}$ are given by equations (17) and (18), respectively. Thus, from above, the criterion of entering and leaving variables is obtained.

\section{Algorithm for solving piece-wise indefinite quadratic programming problem}

Step 1: Consider a piece-wise indefinite quadratic programming problem (PIQPP).

Step 2: Find an initial basic feasible solution $X_{B^{*}}^{0}$ of $(P I Q P P)$.

Step 3: Check the optimality conditions for $X_{B^{*}}^{0}$. If $\xi_{i}^{-} \leq 0$ and $\xi_{i}^{+} \geq 0 ; \forall i=1,2, \ldots p$, then $X_{B^{*}}^{0}$ is an optimal solution. Otherwise, go to step 4 or step 5 according as $\xi_{i}^{+}<0$ or $\xi_{i}^{-}>0$ for some $i$.

Step 4: Let $\xi_{r}^{+}<0$. Choose a non-basic variable $x_{r}$ as the entering variable. Evaluate $\phi_{r}^{*}=\min \left\{\eta_{1}, \eta_{2}, \eta_{3}\right\}$ as given in equation (16). Accordingly, calculate a new basic feasible solution $(\hat{X})^{*}$ as

$$
\left.\begin{array}{l}
\left(\hat{x}_{j}\right)_{r}=\left(x_{j}^{*}\right)_{r}-y_{j}^{*} \phi_{r}^{*}, \forall j \in I^{*} \\
\left(\hat{x}_{r}\right)=x_{r}^{0}+\varphi_{r}^{*} \\
\left(\hat{x}_{j}\right)_{r}=\left(x_{j}^{0}\right), j \in N^{*} \backslash\{r\}
\end{array}\right\}
$$


Go to step 6.

Step 5: Let $\xi_{r}^{-}>0$. Then, a non-basic variable $x_{r}$ is chosen as the entering variable. Compute $\phi_{r}^{*}=\min \left\{\eta_{1}, \eta_{2}, \eta_{3}\right\}$ as in equation (19). Enumerate a new basic feasible criteria $(\hat{X})^{*}$ as

$$
\left.\begin{array}{l}
\left(\hat{x}_{j}\right)_{r}=\left(x_{j}^{*}\right)_{r}+y_{j r}^{*} \phi_{r}^{*}, \forall j \in I^{*} \\
\left(\hat{x}_{r}\right)=x_{r}^{0}+\varphi_{r}^{*} \\
\left(\hat{x}_{j}\right)_{r}=x_{j}^{0}, j \in N^{*} \backslash\{r\}
\end{array}\right\}
$$

Go to step 6 .

Step 6: Update the new basis and examine the optimality condition for this new basic feasible condition. If the obtained new solution is optimal, then it is an optimal solution for (PIQPP). Otherwise go to step 4 or step 5 accordingly.

\section{An illustrative example}

In this section, algorithm is explained by a numerical example. Suppose an investor wants to invest a particular amount of money in the market.He surveyed three companies A, B and $\mathrm{C}$ which offer interest and loyalty additions to its lenders. Let $x_{1}, x_{2}$, and $x_{3}$ be the time (in months) which an investor can consider to be with company $\mathrm{A}, \mathrm{B}$ and $\mathrm{C}$ respectively. Company A offers interest to its lenders represented by the function $Z_{11}\left(x_{1}\right)$. Also, it provides loyalty additions represented by the function $Z_{12}\left(x_{1}\right)$. Similarly, company B offers interest represented by the function $Z_{21}\left(x_{2}\right)$ and loyalty additions represented by the function $Z_{22}\left(x_{2}\right)$. Likewise, company $\mathrm{C}$ has $Z_{31}\left(x_{3}\right)$ and $Z_{32}\left(x_{3}\right)$ as its interest and loyalty addition functions respectively.The maximum period for which one can invest money in company $\mathrm{A}, \mathrm{B}$ and $\mathrm{C}$ is 6, 4 and 6 months respectively. The following are the time constraints for the investor:

$$
\begin{aligned}
2 x_{1}+3 x_{2}+x_{3} & =15 \\
x_{1}+x_{2}+x_{3} & =10
\end{aligned}
$$

The objective is to find how long an investor should invest his money in three companies to maximize his profit. (All amounts in million rupees)

$$
\begin{aligned}
& Z_{11}\left(x_{1}\right)=\left\{\begin{array}{l}
3 x_{1}+0,0 \leq x_{1} \leq 2 \\
5 x_{1}-4,2 \leq x_{1} \leq 6
\end{array}\right. \\
& Z_{12}\left(x_{1}\right)= \begin{cases}-x_{1}+5,0 \leq x_{1} \leq 2 \\
3 x_{1}-3,2 \leq x_{1} \leq 6\end{cases} \\
& Z_{21}\left(x_{2}\right)= \begin{cases}2 x_{2}+5,0 \leq x_{2} \leq 2 \\
6 x_{2}-3,2 \leq x_{2} \leq 4\end{cases} \\
& Z_{22}\left(x_{2}\right)= \begin{cases}-x_{2}+3,0 \leq x_{2} \leq 2 \\
x_{2}-1, & 2 \leq x_{2} \leq 4\end{cases} \\
& Z_{31}\left(x_{3}\right)= \begin{cases}x_{3}+4, & 0 \leq x_{3} \leq 2 \\
2 x_{3}+2,2 \leq x_{3} \leq 3 \\
3 x_{3}-1, & 3 \leq x_{3} \leq 6\end{cases} \\
& Z_{32}\left(x_{3}\right)= \begin{cases}-2 x_{3}+5,0 \leq x_{3} \leq 2 \\
-x_{3}+3, & 2 \leq x_{3} \leq 3 \\
x_{3}-3, & 3 \leq x_{3} \leq 6\end{cases}
\end{aligned}
$$


Solution. The piece-wise indefinite quadratic programming is defined as

$$
\operatorname{Max} \sum_{i=1}^{3} Z_{i}\left(x_{i}\right)
$$

subject to

$$
\begin{gathered}
2 x_{1}+3 x_{2}+x_{3}=15 \\
x_{1}+x_{2}+x_{3}=10 \\
0 \leq x_{1} \leq 6,0 \leq x_{2} \leq 4,0 \leq x_{3} \leq 6
\end{gathered}
$$

where $Z_{i}\left(x_{i}\right)=Z_{i 1}\left(x_{i}\right) \cdot Z_{i 2}\left(x_{i}\right) ; i=1,2,3$.

Introducing artificial variables $x_{4}$ and $x_{5}$, problem (20) can be written as

$$
\operatorname{Max} \sum_{i=1}^{5} Z_{i}\left(x_{i}\right)
$$

subject to

$$
\begin{array}{r}
2 x_{1}+3 x_{2}+x_{3}+x_{4}=15 \\
x_{1}+x_{2}+x_{3}+x_{5}=10
\end{array}
$$

$0 \leq x_{1} \leq 6,0 \leq x_{2} \leq 4,0 \leq x_{3} \leq 6, x_{4} \geq 0, x_{5} \geq 0$

where $Z_{4}\left(x_{4}\right)=\left(-M \cdot x_{4}+0\right)\left(0 . x_{4}+0\right)$

$Z_{5}\left(x_{5}\right)=\left(-M . x_{5}+0\right)\left(0 . x_{5}+0\right) ; M>0$

The initial table for the problem is shown in Table 1.

\begin{tabular}{|lcccccccc|}
\hline$C_{B}$ & $D_{B}$ & $V_{B}$ & $X_{B}$ & $x_{1}$ & $x_{2}$ & $x_{3}$ & $x_{4}$ & $x_{5}$ \\
\hline \hline$-M$ & 0 & $x_{4}$ & 15 & 2 & 3 & 1 & 1 & 0 \\
$-M$ & 0 & $x_{5}$ & 10 & 1 & 1 & 1 & 0 & 1 \\
\hline$Z_{1}=9-25 M$ & & & $\xi_{i}^{+}$ & $-\frac{15}{2}-\frac{83}{2} M$ & $-7-57 \mathrm{M}$ & $25-36 \mathrm{M}$ & 0 & 0 \\
$Z_{2}=13$ & & & $\xi_{i}^{-}$ & 0 & 0 & 0 & 0 & 0 \\
\hline
\end{tabular}

Table 1: Initial solution

Select $x_{1}$ as an entering variable. For departing variable, calculate $\phi_{r}^{*}=\operatorname{Min}\left(\eta_{1}, \eta_{2}, \eta_{3}\right)$, that is, $\phi_{r}^{*}=\operatorname{Min}\left(\frac{15}{2}, \frac{10}{1}, 2\right)$. Now, $\phi_{r}^{*}=2$, corresponding to $\eta_{3}$, therefore, $x_{1}$ will increase from 0 to 2 , but it will continue as a non-basic variable. Accordingly, $x_{4}=11, x_{5}=8$. The next basic table is shown in Table 2 .

\begin{tabular}{|lcccccccc|}
\hline$C_{B}$ & $D_{B}$ & $V_{B}$ & $X_{B}$ & $x_{1}$ & $x_{2}$ & $x_{3}$ & $x_{4}$ & $x_{5}$ \\
\hline \hline$-M$ & 0 & $x_{4}$ & 11 & 2 & 3 & 1 & 1 & 0 \\
$-M$ & 0 & $x_{5}$ & 8 & 1 & 1 & 1 & 0 & 1 \\
\hline$Z_{1}=15-19 M$ & & & $\xi_{i}^{+}$ & $-365-\frac{51}{2} M$ & $\frac{1}{3}-\frac{145}{3} M$ & $35-48 \mathrm{M}$ & 0 & 0 \\
$Z_{2}=11$ & & & $\xi_{i}^{-}$ & 0 & 0 & 0 & 0 & 0 \\
\hline
\end{tabular}

Table 2: Revised solution 


\begin{tabular}{|lcccccccc|}
\hline$C_{B}$ & $D_{B}$ & $V_{B}$ & $X_{B}$ & $x_{1}$ & $x_{2}$ & $x_{3}$ & $x_{4}$ & $x_{5}$ \\
\hline \hline 3 & 1 & $x_{3}$ & 5 & 0 & 1 & -1 & 1 & -2 \\
5 & 3 & $x_{1}$ & 5 & 1 & 1 & 1 & 0 & 1 \\
\hline$Z_{1}=40$ & & & $\xi_{i}^{+}$ & 0 & 152 & 0 & $76+12 M$ & $28+12 M$ \\
$Z_{2}=11$ & & & $\xi_{i}^{-}$ & 0 & 0 & 0 & 0 & 0 \\
\hline
\end{tabular}

Table 3: Optimal solution

From Table 2, select $x_{1}$ as an entering variable. Calculate $\phi_{r}^{*}=\operatorname{Min}\left(\frac{11}{2}, \frac{8}{1}, 4\right) . x_{1}$ will increase its value to 6 and the basis will remain same. The new solution is given by $(6,0,0,0,0)$. Proceeding according to the algorithm, the optimal table is given by table 3 . From Table 3 $\xi_{i}^{-} \leq 0$ and $\xi_{i}^{+} \geq 0 ; i=1,2,3,4,5$. Therefore, optimality condition is satisfied. Hence, the optimal solution for piece-wise indefinite quadratic programming problem is $(5,0,5,0,0)$ with $\operatorname{Max} Z=680$. Here, $x_{1}=5, x_{2}=0, x_{3}=5$. This means that a person should invest his money with company $\mathrm{A}$ and $\mathrm{C}$ for 5 months and with company $\mathrm{B}$, he should not invest at all. His profit from investing money in three companies is $R s .680$ million. The above example is also solved using computing software LINGO 17.0. The optimal solution obtained is $(5,0,5,0,0)$ which is same as the solution obtained from the algorithmic approach.

\section{Conclusion}

In this paper, a novel idea is proposed to evaluate piece-wise indefinite quadratic programming problem. This approach considers those objective functions which are quasi-concave. A proposition has been proved exhibiting the conditions where sum of quasi-concave functions will be quasi-concave. An algorithm is developed moving from one basic feasible solution to another basic feasible solution, thus, ultimately reaching to the optimal solution. A numerical example from real world is formulated demonstrating the algorithm and is solved using LINGO 17.0 software. The results so derived computationally have also verified the algorithmic approach.

This paper scrutinizes piece-wise indefinite quadratic programming problems due to its relevance. piece-wise indefinite quadratic programming can be applied on the function of return on investment where we want to maximize the profit on number of shares of a company bought and dividends earned thereon. The number of shares bought depends upon a particular company and the amount which one wants to invest.

\section{Acknowledgements}

The author is grateful to the editor(s), and would like to thank the reviewers for their valuable suggestions and comments.

\section{References}

[1] Aneja Y. P., Aggarwal, V. and Nair, K. P. K. (1984). On a class of quadratic programs. European Journal of Operational Research, 18(1), 62-70. doi: 10.1016/0377-2217(84)90262-5

[2] Benchekroun, B. and Falk, J. E. (1991). A non-convex piece-wise linear optimization problem. Computers and Mathematics with Applications, 21(6-7), 77-85. doi: 10.1016/0898-1221(91)90162$\mathrm{W}$

[3] Cabot, A.V. (1978). Maximizing the sum of certain quasiconcave functions using generalized benders decomposition. Naval Research Logistics Quarterly, 25(3), 473-481. doi: 10.1002/nav.3800250309

[4] Chang, C. T. (2002). A modified goal programming model for piece-wise linear functions. European Journal of Operational Research, 139(1), 62-67. doi: 10.1016/S0377-2217(01)00178-3 
[5] Chen, M. J. and Huang, G. H. (2001). A derivative algorithm for inexact quadratic programapplication to environmental decision making under uncertainty. European Journal of Operational Research, 128(3), 570-586. doi: 10.1016/S0377-2217(99)00374-4

[6] Fourer, R. (1985). A simplex algorithm for piece-wise linear programming I: Derivation and proof. Mathematical Programming, 33(2), 204-233. doi: 10.1007/BF01582246

[7] Fourer, R. (1992). A simplex algorithm for piece-wise linear programming III: Computational analysis and applications. Mathematical Programming, 53(1-3), 213-235. doi: 10.1007/BF01585703

[8] Ge, D., Wan, G., Wang, Z. and Zhang, J. (2013). A note on appointment scheduling with piece-wise linear cost functions. Mathematics of Operations Research, 39(4), 1244-1251. doi: $10.1287 /$ moor.2013.0631

[9] Keha, A. B., de Farias, I. R. and Nemhauser, G. L. (2004). Models for representing piece--wise linear cost functions. Operations Research Letters, 32(1), 44-48. doi: 10.1016/S0167-6377(03)00059-2

[10] Kough, P.F. (1979). The indefinite quadratic programming problem. Operations Research, 27(3), 516-533. doi: 10.1287/opre.27.3.516

[11] Murty, K. G. (1983). Linear programming, John Wiley and Sons.

[12] Murty, R. V. and Helgason, R. V. (1994). A direct simplex algorithm for network flow problem with convex piece-wise linear costs. Optimization Methods and Software, 4(3), 191-207. doi: $10.1080 / 10556789408805587$

[13] Pandey, P. and Punnen, A. P. (2007). A simplex algorithm for piece-wise-linear fractional programming problems. European Journal of Operational Research, 178(2), 343-358. doi: 10.1016/j.ejor.2006.02.021

[14] Pardalos, P. M., Glick, J. H. and Rosen, J. B. (1987). Global minimization of indefinite quadratic problems. Computing, 39(4), 281-291. doi: 10.1007/BF02239972

[15] Shi, Y., Peng, Y., Kou, G. and Chen, Z. (2005). Classifying credit card accounts for business intelligence and decision making: A multiple-criteria quadratic programming approach. International Journal of Information Technology and Decision Making, 4(4), 581-599. doi: $10.1142 / \mathrm{S} 0219622005001775$ 